July 2006

\title{
Comparison of dynamic (brush) and static (pressure) mechanical allodynia in migraine
}

\author{
C. LoPinto \\ New York University \\ W. B. Young \\ Thomas Jefferson University \\ Avi Ashkenazi \\ Thomas Jefferson University
}

Follow this and additional works at: https://jdc.jefferson.edu/neurologyfp

Part of the Neurology Commons

Let us know how access to this document benefits you

\section{Recommended Citation}

LoPinto, C.; Young, W. B.; and Ashkenazi, Avi, "Comparison of dynamic (brush) and static (pressure) mechanical allodynia in migraine" (2006). Department of Neurology Faculty Papers. Paper 3.

https://jdc.jefferson.edu/neurologyfp/3

This Article is brought to you for free and open access by the Jefferson Digital Commons. The Jefferson Digital Commons is a service of Thomas Jefferson University's Center for Teaching and Learning (CTL). The Commons is a showcase for Jefferson books and journals, peer-reviewed scholarly publications, unique historical collections from the University archives, and teaching tools. The Jefferson Digital Commons allows researchers and interested readers anywhere in the world to learn about and keep up to date with Jefferson scholarship. This article has been accepted for inclusion in Department of Neurology Faculty Papers by an authorized administrator of the Jefferson Digital Commons. For more information, please contact: JeffersonDigitalCommons@jefferson.edu. 


\section{Comparison of dynamic (brush) and static (pressure) mechanical allodynia in migraine}

\section{LoPinto, ${ }^{1}$ WB Young ${ }^{2} \&$ A Ashkenazi ${ }^{2}$}

${ }^{1}$ New York University School of Medicine, New York, NY and ${ }^{2}$ Thomas J efferson University, Jefferson Headache Center, Department of Neurology, Philadelphia, PA, USA

Avi Ashkenazi MD, J efferson Headache Center, Thomas Jefferson University Hospital, 111 South 11th Street, Suite 8130, Philadelphia, PA 19107, USA. Tel. + 1215955 2032, fax + 1215955 2060, e-mail avi.ashkenazi@jefferson.edu

\section{Abstract}

Allodynia has been described in migraine but has not been fully investigated for the different sensory modalities. The aim of this study was to compare the prevalence of dynamic (brush) and static (pressure) mechanical allodynia in migraine patients and to suggest a practical method of testing them in a clinical setting. Patients with International Headache Society-defined episodic migraine (EM) or with transformed migraine (TM) as defined by Silberstein and Lipton were prospectively recruited from the Jefferson Headache Center out-patient clinic. A questionnaire of migraine features and symptoms of allodynia was administered. Brush allodynia (BA) was tested by cutaneous stimulation with a 
gauze pad and pressure allodynia (PA) was tested using von Frey hairs (VFH). The prevalence of BA and PA in all patients and in the different subgroups was calculated and correlated with migraine features. We recruited 55 migraine patients. Twenty-five had EM and 30 had TM. BA was present in $18(32.7 \%)$ patients and PA in 18-24 (32.7-43.6\%). Allodynia to both brush and pressure was found in $13-17(23.6-30.9 \%)$ patients. If a patient had allodynia to one modality only, it was more likely to be PA than BA. Both BA and PA were more common in patients with TM compared with those with EM [BA $46.7 \%$ vs. 16.0\%; PA (differences significant for the medium and thick VFHs) $50 \%$ vs. $20 \%$ and $50 \%$ vs. $12 \%$, respectively]. Both types of allodynia were also more common in patients with migraine with aura compared with those with migraine without aura (BA $57.1 \%$ vs. $17.6 \%$; PA $57.1-61.9 \%$ vs. $17.6-32.7 \%$ ). There was a positive correlation between allodynia score (as obtained by examination) and allodynia index (as obtained by history) for both BA and PA. The incomplete, although considerable, overlap between BA and PA suggests that allodynia to different sensory modalities is associated with sensitization of different neuronal populations. Because PA was more common than BA, it may be a more sensitive indicator of allodynia in migraine. PA can be tested clinically in a practical and systematic manner.

\section{I ntroduction}

Allodynia is a phenomenon in which normally non-painful stimuli are perceived as painful. Cutaneous allodynia is present in approximately $80 \%$ of migraine 
patients during an acute attack (1). There are four types of cutaneous allodynia:

(i) dynamic mechanical (brush), (ii) static mechanical (pressure), (iii) heat and (iv) cold. Allodynia in migraine has not been fully investigated for the different sensory modalities. Burstein et al. investigated heat, cold and static, but not dynamic, mechanical allodynia $(1,2)$. There is evidence that allodynia in migraine is associated with a poor response to triptans (3). Therefore, identifying and using the optimal method to test migraine patients for allodynia is of clinical value. In this study, we compared the prevalence of brush and pressure allodynia in migraine patients. We also propose a systematic and practical method to test patients for allodynia and suggest the use of a brief questionnaire to determine the presence of allodynia in migraine patients.

\section{Methods}

This study was approved by the Institutional Review Board for Studies with Human Subjects of Jefferson University Hospital. All patients signed a document of informed consent prior to enrolment in the study.

Patients with International Headache Society (HIS)-defined episodic migraine (EM) or transformed migraine (TM) as defined by Silberstein and Lipton were prospectively recruited from the Jefferson Headache Center out-patient clinic (4-6). Briefly, the criteria for TM were: (i) headache for $>15$ days/ month for $>1$ month; (ii) average headache duration of $>4 \mathrm{~h} /$ day (untreated); (iii) at least one of the following: (a) history of IHS-defined episodic migraine, (b) history of increasing headache frequency with decreasing severity, (c) current headache meets IHS criteria for migraine other than duration; (iv) exclusion of 
secondary causes of headache (5). Control subjects were recruited to determine cut-off values for pressure allodynia. These were spouses and relatives of patients, recruited from the clinic's waiting room, who did not suffer from migraine headaches. Exclusion criteria for both groups were the presence of peripheral neuropathy or other neurological diseases that may affect sensory function, dermatological disease that may affect skin sensation, secondary headaches (e.g. headache resulting from Lyme disease, post-traumatic headache) or a diagnosis of new daily persistent headache. Patients were allowed to be on headache-preventive medications while participating in the study. A questionnaire of demographic data, migraine history, migraine attack features and symptoms of allodynia was administered. A seven-point allodynia index was determined by the results of the questionnaire (Table 1 ).

Migraine patients were tested for both static mechanical (pressure) and dynamic mechanical (brush) cutaneous allodynia. Brush allodynia (BA) was tested by gently applying a folded $4 \times 4$-in gauze pad to the skin, 10 times at a frequency of $2 / \mathrm{s}$ as we previously described (7). Pressure allodynia (PA) was tested by applying von Frey hairs (VFH) (Touch-Test ${ }^{\mathrm{TM}}$ Sensory Evaluators; North Coast Medical, Inc. Morgan Hill, CA, USA) of three different weights, 1.4 $\mathrm{g}, 8 \mathrm{~g}$ and $60 \mathrm{~g}$, three times to each area, holding down for $1 \mathrm{~s}$ and releasing. Since there are no standardized measures for the amount of pressure that causes pain in normal subjects, the selection of these weights was somewhat arbitrary. The skin areas tested for both BA and PA were the forehead (V1), posterior neck $(\mathrm{C} 2, \mathrm{C} 3)$ and inner forearm (C8), bilaterally. The degree of pain 
or unpleasant sensation upon stimulation was measured using a $100-\mathrm{mm}$ visual analogue scale (VAS).

A potential confounder in the comparison of the two modalities is the subjective distinction between pressure and pain. While any pain sensation resulting from light cutaneous stimulation with a gauze pad is defined as allodynia, some patients may have difficulty in the distinction between a sensation of pressure and pain. To define PA, we used the control group. A number of control subjects did report pain in response to VFH application. To classify a subject's response as allodynic in a standardized manner, the cut-off value for allodynia was defined as the pain level on the VAS below which $95 \%$ of control subjects responded. These cut-off values were $5 \mathrm{~mm}, 34 \mathrm{~mm}$ and $43 \mathrm{~mm}$ for the 1.4-g, 8-g and 60-g VFHs, respectively.

An allodynia score was determined as the sum of allodynia levels as determined by VAS at the different sites. This score was correlated with head pain level at the time of testing. The prevalence of BA and PA was determined for all the patients, as well as for each group (EM and TM) separately.

Data were analysed for the following subgroups: patients with and without BA, patients with or without PA to thin, medium or thick VFHs. Fischer's exact test was used to compare the frequencies of categorical variables such as diagnosis. Wilcoxon scores were used to compare disease duration within these subgroups. Pearson correlation coefficients were calculated to determine correlations between allodynia scores and allodynia indices, as well as head pain severity. 


\section{Results}

We recruited 55 migraine patients (43 women, 12 men, mean age $42.9 \pm 14.4$ years) and 21 non-migraine control subjects (13 women, eight men, mean age $43.6 \pm 16.4$ years). Twenty-five patients had EM and 30 had TM. Average disease duration in the entire study population was 21 years, with no significant difference between patients with EM and those with TM (possibly due to a selection bias of episodic migraine patients who attend a tertiary headache clinic). Average frequency of acute attacks was $2.3 /$ month in the EM group and $4.6 /$ month in the TM group. Seven patients were tested during an acute migraine attack. Six of these patients had allodynia, with five having both BA and PA and one having only PA. The mean time interval between attack onset and allodynia testing was $23 \mathrm{~h}$ (range $2-72 \mathrm{~h}$ ). Of those patients who were tested during an acute attack, two had taken acute migraine drugs less than $2 \mathrm{~h}$ prior to testing [one took dihydroergotamine (DHE) and one took zolmitriptan]. The patient who had taken DHE had PA only, while the patient who had taken zolmitriptan experienced allodynia to both modalities.

Overall, the prevalence of PA was higher than that of BA. BA was present in $18(32.7 \%)$ patients and $\mathrm{PA}$ in $18-24(32.7-43.6 \%)$, depending on the weight of the VFH applied (Fig. 1). If a patient had allodynia to one modality only, it was more likely to be PA than BA (Fig. 2a-c). Both BA and PA were more common in TM compared with EM [BA $46.7 \%$ vs. $16.0 \%, \mathrm{P}=0.02 ; \mathrm{PA}$ (differences significant for the medium and thick VFHs) $50 \%$ vs. $20 \%, P=0.03$ and $50 \%$ vs. $12 \%, P=0.004$, respectively] (Fig. 3). Both types of allodynia were 
also more common in patients with migraine with aura (MA) compared with those with migraine without aura (MoA) $[\mathrm{BA} 57.1 \%$ vs. $17.6 \%, \mathrm{P}=0.003$; PA (data shown for thin hairs) $61.9 \%$ vs. $32.7 \%, \mathrm{P}=0.3$ ] (Fig. 4 ).

There was a positive correlation between allodynia score (obtained by examination) and allodynia index (obtained by history) for both BA (0.39, $P=0.003)$ and PA $(0.39-0.40, P<0.003)$ (Table 2$).$ There was also a positive correlation between allodynia score and headache level at time of testing for both BA (0.56, P $<0.001)$ and PA $(0.43-0.50, \mathrm{P}<0.0007)$ (Table 2). We found no statistically significant correlation between disease duration and the score of either BA or PA.

\section{Discussion}

Using cut-offs based on a control population of non-migraine subjects, we were able to differentiate between migraine patients with and without PA. The use of VFHs may be useful to detect PA in migraine patients in a clinical setting. Triptan therapy for migraine has little efficacy once allodynia has occurred (3). Therefore, having a practical tool to assess this phenomenon will aid in choosing treatment.

Allodynia is thought to arise from central sensitization of second- and third-order neurons in the trigeminal nucleus caudalis (TNC) or thalamus (1). Nociceptive input from dural vessels during a migraine attack may activate the secretion of inflammatory intermediates and neurotransmitters that promote neuronal hyperexcitability. Central sensitization is associated with activation of glutamate and substance $P$ receptors $(8,9)$. These can then activate secondary 
messengers such as protein kinase $C$ and protein kinase $A(8)$. The pattern of allodynia would therefore correspond to higher order representations of sensory input.

PA was more prevalent than BA in this migraine patient group. This indicates that PA may be a more sensitive measure of central sensitization during migraine than BA. There was a considerable, but not complete, overlap between the PA and BA groups, regardless of the weight of VFH used. This suggests that PA and $\mathrm{BA}$ in migraine result from central sensitization of similar, but not identical, neuronal populations. It also suggests that there are separate pathways through which sensitization to brush and to pressure stimuli occur.

Both PA and BA were more common in patients with TM than in those with EM. In a previous study, we demonstrated a high prevalence of BA in TM both during baseline headaches and during headache exacerbations (10). The prevalence of BA in EM in that study was lower than that of TM, but increased significantly when EM patients were tested during an acute attack. The findings of the current study are consistent with these results and suggest that migraine patients with little or no headache are unlikely to have allodynia.

The higher prevalence of allodynia in patients with TM suggests that allodynia may result from a process of repeated neuronal sensitization in the TNC over time. It is of interest to note that similar results were found in cluster headache $(\mathrm{CH})$ patients: the prevalence of BA in these patients was higher in chronic, compared with episodic $\mathrm{CH}$, further supporting the above notion (11). In this study, however, we found no significant difference in disease 
duration between patients with allodynia and those without allodynia. This may be due to the small number of patients.

Both BA and PA were more common in MA compared with MoA in this study. There is no satisfactory explanation to this interesting finding. It suggests, however, that in patients with MA there is a lower threshold for neuronal sensitization in the TNC compared with that of patients with MoA. This hypothesis needs further study.

Finally, our study demonstrates that self-reporting of allodynic symptoms correlates with the presence of allodynia upon testing. This confirms the usefulness of the seven-point questionnaire in rapidly assessing the presence of allodynia in the clinic.

Patients in this study were allowed to remain on migraine-preventive drugs. This may have had an effect on our results since some migrainepreventive drugs (e.g. gabapentin) have been shown to reduce allodynia (12). The use of acute migraine drugs was also allowed in the study, but only two patients had used them less than $2 \mathrm{~h}$ prior to allodynia testing. Sumatriptan has been shown to cause transient allodynia in migraine patients, but this effect was short lasting ( $<40 \mathrm{~min}$ ) (13). The effect of acute migraine drugs on the results of allodynia testing in this studyis therefore not likely to be significant. Another limitation of this study is that patients with EM and those with TM were not analysed separately. Although data on the occurrence of cutaneous allodynia in TM are sparse, patients with TM and those with EM may differ as regards central sensitization and the occurrence of cutaneous allodynia. Further studies are 
needed to examine the value of PA measurement in migraine, to analyse its occurrence in EM and in TM separately and to correlate it with treatment outcome.

\section{References}

1. Burstein R, Yarnitsky D, Goor-Aryeh I, Ransil BJ, Bajwa ZH. An association between migraine and cutaneous allodynia. Ann Neurol 2000; 47:614-24.

2. Burstein R, Cutrer MF, Yarnitsky D. The development of cutaneous allodynia during a migraine attack clinical evidence for the sequential recruitment of spinal and supraspinal nociceptive neurons in migraine. Brain 2000; 123:1703-9.

3. Burstein R, Collins B, Jakubowski M. Defeating migraine pain with triptans: a race against the developing allodynia. Ann Neurol 2004; 55:19-26.

4. Headache Classification Committee. The International Classification of Headache Disorders, 2nd Edition. Cephalalgia 2004;24:1-160.

5. Silberstein SD, Lipton RB, Sliwinski M. Classification of daily and near-daily headaches: field trial of revised IHS criteria. Neurology $1996 ; 47: 871-5$.

6. Lipton RB, Bigal ME, Steiner TJ, Silberstein SD, Olesen J. Classification of primary headaches. Neurology 2004; 63:427-35.

7. Ashkenazi A, Young WB. The effects of greater occipital nerve block and trigger point injection on brush allodynia and pain in migraine. Headache $2005 ; 45: 350-4$.

8. Kawasaki $\mathrm{Y}$, Kohno $\mathrm{T}$, Zhuang ZY, Brenner GJ, Wang $\mathrm{H}$, Meer $\mathrm{C}$ et al. Ionotropic and metabotropic receptors, protein kinase $A$, protein kinase $C$, 
and Src contribute to C-fiber-induced ERK activation and CAMP response element-binding protein phosphorylation in dorsal horn neurons, leading to central sensitization. J Neurosci 2004; 24:8310-21.

9. Khasabov SG, Rogers SD, Ghilardi JR, Peters CM, Mantyh PW, Simone DA. Spinal neurons that possess the substance $\mathrm{P}$ receptor are required for the development of central sensitization. J Neurosci 2002; 22:9086-98.

10. Ashkenazi A, Sholtzow M, Young WB. Prevalence of mechanical (brush) allodynia in migraine. Neurology 2004; 62:A83-A84 (Abstract).

11. Ashkenazi A, Young WB. Dynamic mechanical (brush) allodynia in cluster headache. Headache 2004; 44:1010-2.

12. Berry JD, Petersen KL. A single dose of gabapentin reduces acute pain and allodynia in patients with herpes zoster. Neurology 2005; 65:444-7.

13. Linde M, Elam M, Lundblad L, Olausson H, Dahlof CG. Sumatriptan (5HT1B/1D-agonist) causes a transient allodynia. Cephalalgia 2004; 24:105766. 


\section{TABLES \& FI GURES}

Table 1 A questionnaire to assess symptoms of allodynia. Allodynia index is the sum of positive answers

Is your headache made worse by:

Tilting or bending down your head

Coughing

Climbing stairs
( ) yes; ( ) no

( ) yes; ( ) no

( ) yes; ( ) no

Do any of the following bother you when you have a headache:

Touching your scalp or face

Wearing any object on your head/neck

(hat, jewelry, glasses, necklaces, etc.)

Wearing any object on your arms/wrist

(watch, jewelry, long sleeves, etc.)

Exposure of your face/head to wind
( ) yes; ( ) no

( ) yes; ( ) no

( ) yes; ( ) no

( ) yes; ( ) no 
Table 2 Correlation between allodynia index (obtained by questionnaire) and allodynia scores (obtained by examination) and between headache level at time of testing and allodynia scores

Pearson

correlation

Parameter Modality of allodynia score coefficient P-value $\begin{array}{llll}\text { Allodynia index } & \text { Brush allodynia } & 0.39 & 0.0030\end{array}$

Pressure allodynia (thin hair) $\quad 0.39 \quad 0.0031$

Pressure allodynia (medium hair) $\quad 0.40 \quad 0.0024$

Pressure allodynia (thick hair) $\quad 0.39 \quad 0.0030$

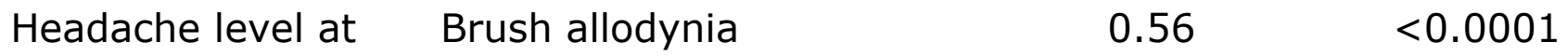

$\begin{array}{llll}\text { time of testing } & \text { Pressure allodynia (thin hair) } & 0.43 & 0.0011\end{array}$

Pressure allodynia (medium hair) $0.50 \quad<0.0001$

$\begin{array}{lll}\text { Pressure allodynia (thick hair) } & 0.43 & 0.0007\end{array}$ 
Figure 1 Prevalence of brush allodynia (BA) and of pressure allodynia (PA) with thin $(1.4 \mathrm{~g})$, medium $(8 \mathrm{~g})$ and thick $(60 \mathrm{~g})$ von Frey hairs (VFHs).

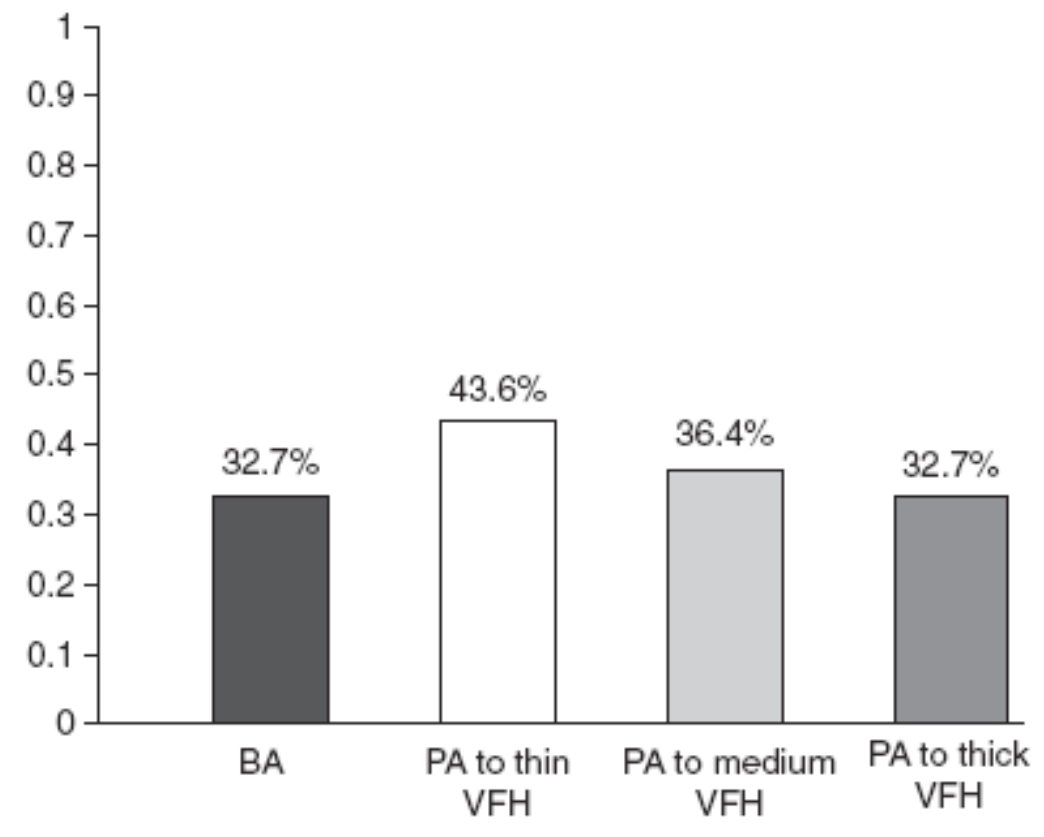

Figure 2 The overlap of brush allodynia (BA) and pressure allodynia (PA) using thin (a), medium (b) and thick von Frey hairs (c) 
a Percent distribution of patients with both PA and

BA, BA only, PA only or nelther for thin VFH

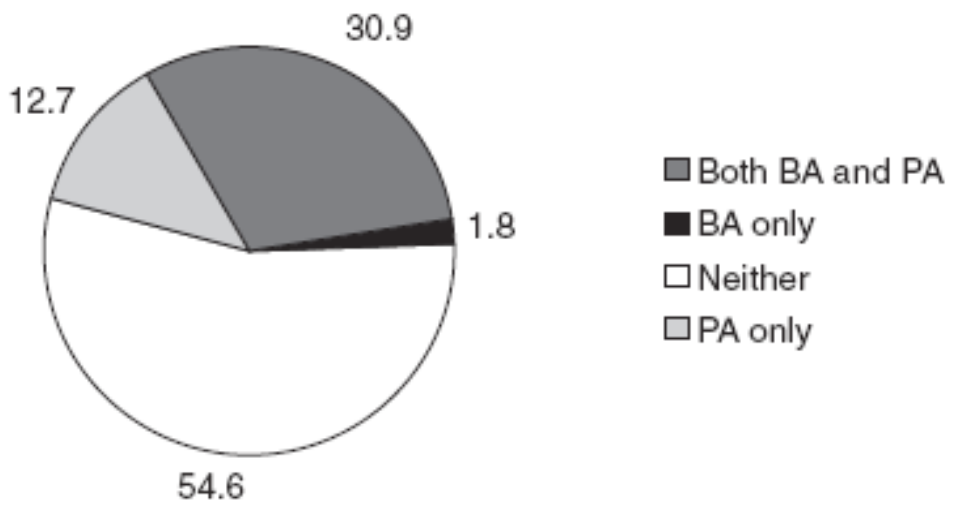

b Percent distribution of patients with both PA and BA, BA only, PA only or nelther for medlum VFH

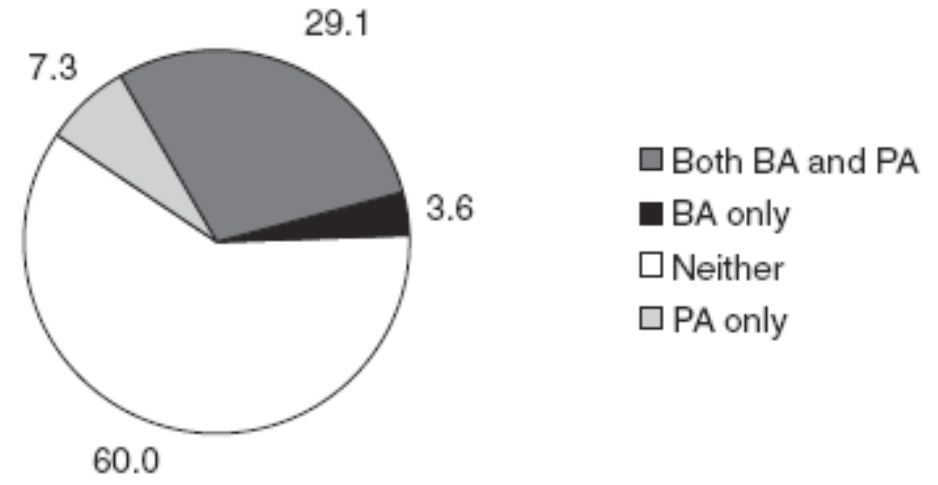

c Percent distribution of patients with both PA and

BA, BA only, PA only or neither for thick VFH

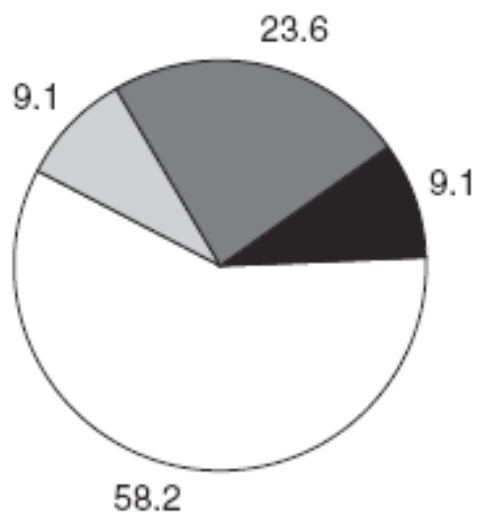

$\square$ Both BA and PA

-BA only

$\square$ Neither

$\square$ PA only 
Figure 3 Prevalence of brush allodynia (BA) and pressure allodynia (PA) in patients with transformed migraine ( $\square$ ) and episodic migraine ( $\square$ ).

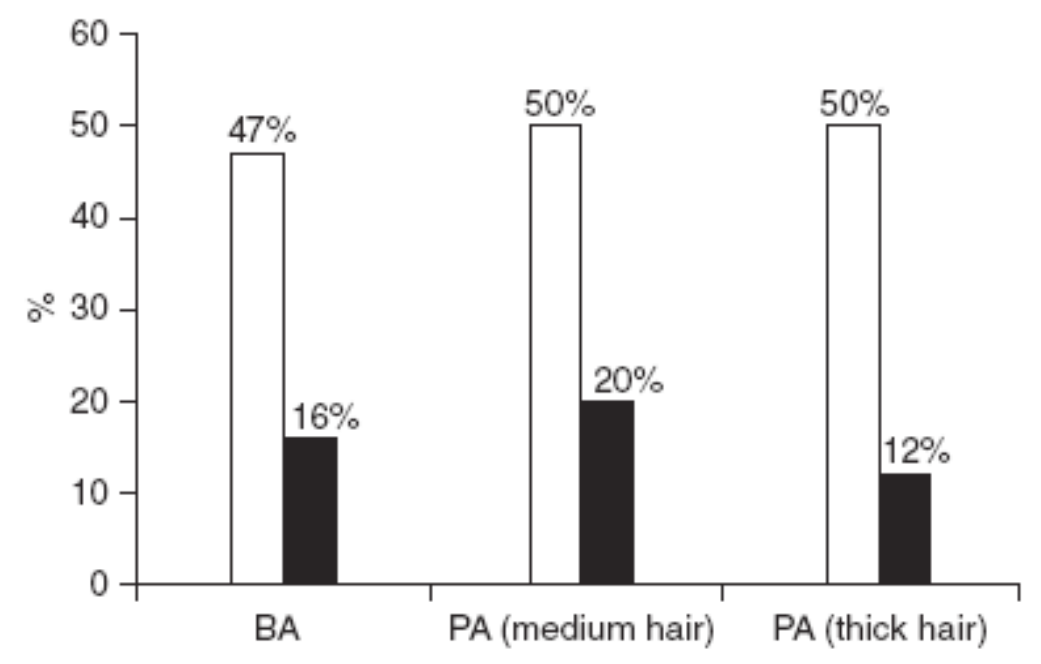

Figure 4 Prevalence of brush allodynia (BA) and pressure allodynia (PA) (for thin von Frey hairs) in patients with migraine with aura $(\square, \mathrm{n}=21)$ and in those with migraine without aura $(\mathbf{a}, \mathrm{n}=34)$.

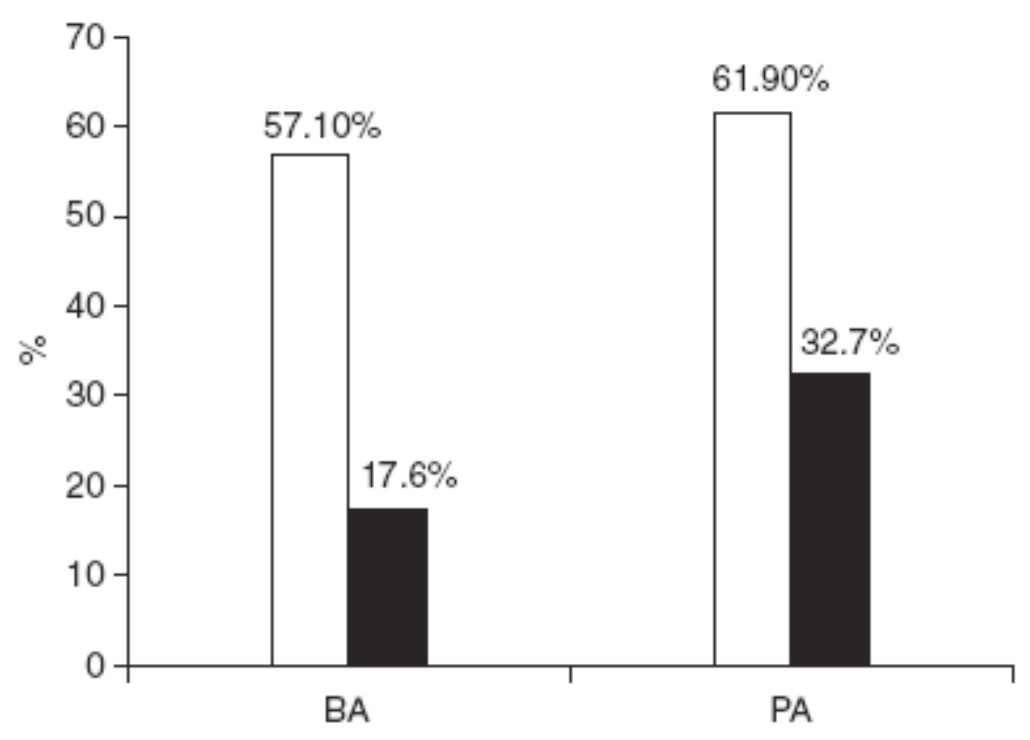

